TITLE:

\title{
Macular choroidal thickness and volume in eyes with angioid streaks measured by swept source optical coherence tomography.
}

\section{AUTHOR(S):}

Ellabban, Abdallah A; Tsujikawa, Akitaka; Matsumoto, Akiko; Ogino, Ken; Hangai, Masanori; Ooto, Sotaro; Yamashiro, Kenji; Akiba, Masahiro; Yoshimura, Nagahisa

\section{CITATION:}

Ellabban, Abdallah A ... [et al]. Macular choroidal thickness and volume in eyes with angioid streaks measured by swept source optical coherence tomography.. American journal of ophthalmology 2012, 153(6): 1133-1143.e1

\section{ISSUE DATE:}

2012-06

URL:

http://hdl.handle.net/2433/156160

\section{RIGHT:}

(c) 2012 Elsevier Inc.; この論文は出版社版でありません。引用の際には 出版社版をご確認ご利用ください。; This is not the published version. Please cite only the published version. 
Elsevier Editorial System(tm) for American Journal of Ophthalmology Manuscript Draft

Manuscript Number: AJ0-11-692R2

Title: Macular Choroidal Thickness and Volume in Eyes with Angioid Streaks Measured by Swept Source Optical Coherence Tomography

Article Type: Original Article

Keywords: Angioid streaks; swept source optical coherence tomography; choroidal thickness; choroidal volume; choroidal neovascular membrane.

Corresponding Author: Dr. Akitaka Tsujikawa, MD

Corresponding Author's Institution: Kyoto University Graduate School of Medicine

First Author: ABDALLAH A ELLABBAN, MD

Order of Authors: ABDALLAH A ELLABBAN, MD; AKITAKA TSUJIKAWA; AKIKO MATSUMOTO; KEN

OGINO, MD; MASANORI HANGAI, MD; SOTARO OOTO, MD; KENJI YAMASHIRO, MD; MASAHIRO AKIBA, PhD; NAGAHISA YOSHIMURA, Professor 


\section{Abstract}

PURPOSE: To study the mean choroidal thickness and volume of the macula in eyes with angioid streaks using swept source optical coherence tomography (OCT) in the $1,050 \mathrm{~nm}$ wavelength range.

DESIGN: Prospective case series.

METHODS: The macular area of 39 eyes of 23 patients with angioid streaks and of 20 normal eyes of 20 matched controls (Group 1) was studied with a swept source OCT prototype system. Eyes with angioid streaks were classified into one of four groups: those without choroidal neovascularization (CNV) (Group 2); those with CNV that had no history of treatment (Group 3); those with CNV that had previously received only anti-vascular endothelial growth factor treatments (Group 4); and those with CNV that had previously received photodynamic therapy (Group 5). Using a raster scan protocol with $512 \times 128$ A-scans, we produced a macular choroidal thickness map $\left(6 \times 6 \mathrm{~mm}^{2}\right)$. RESULTS: There were no significant differences in age, axial length, or refractive error among the five groups. Mean choroidal thickness of the macula in Group $2(218.9 \pm 46.8$ $\mu \mathrm{m})$ was as great as was that in Group $1(218.8 \pm 69.2 \mu \mathrm{m})$. However, the macular choroidal thickness in Group $3(119.7 \pm 49.2 \mu \mathrm{m})$, Group $4(140.1 \pm 64.9 \mu \mathrm{m})$, and Group $5(144.0 \pm 52.6 \mu \mathrm{m})$ was significantly less than that of Group $1(P<.05)$. There were no statistical differences between Groups 3-5. In each group, the choroid of the nasal quadrant was significantly thinner compared to that in other quadrants $(P<.05)$.

CONCLUSIONS: The choroid in eyes with angioid streaks without CNV was as thick as was that in normal controls, but was significantly thinner in eyes with angioid streaks that had developed CNV. 


\title{
Macular Choroidal Thickness and Volume in Eyes with Angioid Streaks Measured by Swept Source Optical Coherence Tomography
}

Short title: Macular Choroidal Thickness in Angioid Streaks

\begin{abstract}
ABDALLAH A. ELLABBAN, ${ }^{1}$ AKITAKA TSUJIKAWA, ${ }^{1}$ AKIKO MATSUMOTO,${ }^{1,2} \mathrm{KEN}$ OGINO, ${ }^{1}$ MASANORI HANGAI, ${ }^{1}$ SOTARO OOTO, ${ }^{1}$ KENJI YAMASHIRO, ${ }^{1}$ MASAHIRO AKIBA, ${ }^{2}$ AND NAGAHISA YOSHIMURA ${ }^{1}$
\end{abstract}

Supplemental material available at AJO.com

From ${ }^{1}$ the Department of Ophthalmology and Visual Sciences, Kyoto University Graduate School of Medicine, Kyoto, Japan; ${ }^{2}$ Topcon Corporation, Tokyo, Japan.

\footnotetext{
Correspondence to Akitaka Tsujikawa, MD, Department of Ophthalmology and Visual Sciences, Kyoto University Graduate School of Medicine, Sakyo-ku, Kyoto 606-8507, Japan; phone: +81-75-751-3260; fax: +81-75-752-0933; e-mail: tujikawa@kuhp.kyoto-u.ac.jp
} 
Pseudoxanthoma elasticum is a rare multisystem disorder associated with a mutation in the $A B C C 6$ gene. ${ }^{1}$ Pseudoxanthoma elasticum causes progressive fragmentation and calcification of elastic fibers in connective tissue, resulting in pathologic changes that are most pronounced in the dermis, blood vessels, and Bruch's membrane. ${ }^{2}$ Characteristic fundus changes of pseudoxanthoma elasticum are peau d'orange, areas of chorioretinal atrophy, and angioid streaks. ${ }^{3,4}$ Angioid streaks are irregular crack-like dehiscences in Bruch's membrane which often allow the ingrowth of choroidal neovascularization (CNV) through Bruch's membrane defects. ${ }^{3,4}$ CNV secondary to angioid streaks occurs in $72-86 \%$ of eyes, is bilateral in more than $70 \%$ of cases, and often occurs at a younger age than does the CNV of age-related macular degeneration (AMD). Because CNV secondary to angioid streaks is often refractory to treatment, it leads in many cases to poor vision if the CNV involves the macular area. ${ }^{5-9}$

Recently, the choroid, which is a structure with one of the highest blood flows in the body, ${ }^{10}$ is reported to be involved in the pathogenesis of various ocular diseases, including AMD,${ }^{11}$ polypoidal choroidal vasculopathy, ${ }^{12}$ central serous chorioretinopathy, ${ }^{13}$ Vogt-Koyanagi-Harada disease,${ }^{14}$ and myopic chorioretinal degeneration. ${ }^{15}$ In angioid streaks, although Bruch's membrane is thought to be most affected, previous reports have suggested that the choroid is also involved in the pathogenesis of CNV. ${ }^{3,4}$ Today, optical coherence tomography (OCT) is used to measure retinal thickness and to detect morphologic changes in various retinal diseases. However, commercially available OCT equipment visualizes the entire choroid only in eyes with high myopia because of low penetration and high backscattering at the level of the retinal pigment epithelium (RPE). ${ }^{16}$, ${ }^{17}$ So, to date, little in vivo information is available on the choroidal changes associated with angioid streaks.

Since Spaide and associates ${ }^{16}$ introduced enhanced depth imaging OCT based on spectral-domain OCT technology, many investigators have studied the choroidal thickness in eyes with various pathologies. ${ }^{11,14,15,18-25}$ Although normal choroidal thickness in the macula varies with region and can vary even more in pathologic conditions, in most of these studies the choroidal thickness was measured at the foveal center or, sometimes, at several measurement points. ${ }^{11,14,15,18-25}$ This is because enhanced depth imaging requires the averaging of 50-100 B-scans of an identical location of interest, ${ }^{16}$ hindering us from high density scanning. With the advances in OCT technology, the recent generation has utilized swept source laser technology, which has the advantage of a high speed scanning rate and low sensitivity roll-off versus depth compared to spectral-domain OCT. ${ }^{26-34}$ With the use of a light source at $1 \mu \mathrm{m}$ wavelength region, swept source OCT allows for a high contrast, high-penetration image of the entire choroid. Based on these advantages, swept source OCT at a longer wavelength allows 
us to obtain a three-dimensional (3D) high contrast image of the choroid.

In the study described herein, we scanned the macular area of eyes with angioid streaks associated with pseudoxanthoma elasticum using swept source OCT at 1,050 $\mathrm{nm}$ with a 3D raster scan protocol, and produced a choroidal thickness map of the macular area. By applying the grid used by the Early Treatment Diabetic Retinopathy Study (ETDRS) to this map, the mean choroidal thickness and volume in each sector was measured and compared with those of normal subjects.

\section{PATIENTS AND METHODS}

This prospective study consisted of 39 eyes of 23 patients with angioid streaks secondary to pseudoxanthoma elasticum. The macular area of these 39 eyes was examined with a swept source OCT prototype system at Kyoto University Hospital between September 2010 and November 2011. The diagnosis of pseudoxanthoma elasticum was based on systemic manifestations, fundus examination, and fluorescein angiography, ${ }^{35}$ and the diagnosis was confirmed by characteristic histopathologic abnormalities in a skin biopsy.

All subjects underwent a thorough ocular examination, including autorefractometry (ARK1; Nidek, Gamagori, Japan), best-corrected visual acuity measurement with a 5-meter Landolt chart, axial length measurement using ocular biometry (IOLMaster; Carl Zeiss Meditec, Jena, Germany), slit lamp examination, intraocular pressure measurement, dilated color fundus photography (TRC50LX; Topcon Corp., Tokyo, Japan), and prototype swept source OCT examination. All eyes with angioid streaks underwent simultaneous fluorescein angiography and indocyanine green angiography using Spectralis HRA+OCT (Heidelberg Engineering, Heidelberg, Germany). Eyes with poor images due to opaque media (e.g., cataracts or vitreous hemorrhage) or to unstable fixation were excluded from the study.

Eligible eyes with angioid streaks were classified into four groups based on the presence of CNV and the previous treatment of the CNV: eyes with angioid streaks without CNV (Group 2), eyes with CNV secondary to angioid streaks that had no history of treatments (Group 3), eyes with CNV secondary to angioid streaks that had previously received only anti-vascular endothelial growth factor (VEGF) treatments with no history of photodynamic therapy (PDT) (Group 4), and eyes with CNV secondary to angioid streaks that did have a history of PDT (Group 5). Data obtained from the four groups with angioid streaks were compared with data obtained from 20 healthy eyes (20 subjects) of controls, matched for age, axial length and refractive error (Group 1). ${ }^{36}$ Exclusion criteria for these 20 controls included history or evidence of chorioretinal or vitreoretinal diseases, 
including AMD, diabetic retinopathy, central serous chorioretinopathy, epiretinal membrane, and macular dystrophy, or a history of intraocular surgery. Subjects with systemic diseases or conditions that might affect retinal or choroidal thickness were also excluded.

\section{SWEPT SOURCE OCT SYSTEM AND SCAN PROTOCOLS}

The prototype swept source OCT system (Topcon Corp.) used in the current study has been reported previously. ${ }^{36}$ In brief, this swept source OCT system utilizes a light source of a wavelength-sweeping laser centered at 1,050 nm with $100,000 \mathrm{~Hz}$ repetition rate. Axial and transverse resolutions were $8 \mu \mathrm{m}$ and $20 \mu \mathrm{m}$ in tissue, respectively. Swept source OCT imaging operated at $1 \mu \mathrm{m}$ wavelength region was conducted at $\sim 1 \mathrm{~mW}$ on the cornea, which is well below the safe retinal exposure limit established by the American National Standards Institute.

Swept source OCT examinations of the eligible subjects were performed by trained examiners after pupil dilation. A 3D imaging data set was acquired on each subject by using a raster scan protocol of 512 (horizontal) $\times 128$ (vertical) A-scans per data set (total 65,536 axial scans/data set) in 0.8 seconds. Each 3D raster scan covered a $6 \times 6-\mathrm{mm}^{2}$ area centered on the fovea, which was confirmed by internal-fixation and by a fundus camera built into the swept source OCT system. To reduce speckle noise, each image was enhanced by weighted moving average of three consecutive original images.

In each subject, 50 averaged horizontal and vertical scan images in $12 \mathrm{~mm}$ transverse scan length were obtained as well. The 50 single images, where each image consisted of 1,024 A-scans, were registered and averaged by software to create an averaged single image. The vertical scan was centered on the fovea while the horizontal scan was centered on the midpoint between the fovea and optic disc.

\section{CHOROIDAL THICKNESS AND VOLUME MEASUREMENT PROTOCOL}

The choroidal thickness was measured as the distance between the outer border of the RPE and the chorioscleral border. In areas where the RPE was defective, the Bruch's membrane was considered as an inner border of the choroid. In each image of the 3D-data set, lines of both RPE and the chorioscleral border were determined manually by a trained observer in a masked fashion. Automated built-in calibration software determined the distance between these two lines. The measurement points per image consisted of 512 points with an interval of $\sim 12 \mu \mathrm{m}$. From the 128 images of each 3D-data set, a choroidal thickness map of $6 \times 6-\mathrm{mm}^{2}$ area was created. After the choroidal thickness map was obtained, the ETDRS grid was applied to the map. ${ }^{37}$

We compared data on the mean choroidal thickness and volumes of the ETDRS 
grid between the five study groups and also compared these values within each group. Using the averaged OCT images, we also performed measurement of retinal thickness and of choroidal thickness at the center of the fovea. Retinal thickness was defined as the distance between the vitreoretinal interface and RPE.

\section{STATISTICAL ANALYSIS}

Statistical analysis was performed using SPSS statistical software (version 16; SPSS Inc., Chicago, Illinois). All values are presented as mean \pm standard deviation. The data were analyzed using one-way analysis of variance with Tukey's post-hoc analysis to compare mean choroidal thickness and volume at different regions. To compare the thickness and volume data in each group, two-way analysis of variance was used. $P$ values less than .05 were considered to be statistically significant.

\section{RESULTS}

In the current study, 39 eyes (23 patients, 14 men and 9 women) with angioid streaks secondary to pseudoxanthoma elasticum, ranging in age from 57 to 80 years (mean: $65.4 \pm 7.4$ years), were examined. Mean refractive error was $-1.19 \pm 2.15$ diopters (range: -5.5 to +1.75 diopters). Mean axial length was $23.83 \pm 1.39 \mathrm{~mm}$ (range: 22.15 to $26.54 \mathrm{~mm}$ ). Eyes with angioid streaks were classified into four groups: those with angioid streaks but with no CNV (Group 2, $n=6$ ), eyes with CNV secondary to angioid streaks with no history of treatments for their CNV (Group 3, $n=7$ ), eyes with CNV secondary to angioid streaks with a history of only anti-VEGF treatments for their CNV (Group 4, $\mathrm{n}=$ 11), and eyes with CNV secondary to angioid streaks with a history of PDT (Group 5, $n=$ 15). Table 1 shows the ocular characteristics of each group. All eyes in Group 4 received anti-VEGF treatments for CNV, with the mean number of injections being $3.4 \pm 2.1$. Eyes in Group 5 received $2.9 \pm 1.9$ PDT treatments and $3.1 \pm 3.9$ anti-VEGF treatments. There were no differences in age, axial length, or refractive error between the five groups.

\section{CHOROIDAL STRUCTURE}

The swept source OCT system operated at $1,050 \mathrm{~nm}$ wavelength range enables visualization of the clear structure of the posterior pole as well as allowing deeper penetration into the choroid. Multi-averaged scans of $12 \mathrm{~mm}$ in length revealed structures of the retina and choroid, which allowed precise identification in all eyes of the chorioscleral border beyond the vascular arcade (left, Figure 1). Figure 2 shows typical cases of each group. In eyes with angioid streaks without CNV (Group 2), the choroid 
was well-preserved and was as thick as was that of healthy controls (Group 1). Multi-averaged scans of the current OCT system often show a layer of medium diameter blood vessels (Sattler's layer) and an outermost layer of the choroid consisting of larger diameter blood vessels (Haller's layer). However, the choroid in eyes with CNV secondary to angioid streaks was remarkably thin with disorganized configuration of choroidal vessels (Groups 3-5), regardless of a history of treatment. In eyes with CNV secondary to angioid streaks that had a history of PDT (Group 5), choroidal thickness was reduced not only in the macular area, where the PDT was performed, but also beyond the vascular arcade.

\section{MEAN CHOROIDAL THICKNESS AND VOLUME}

Using a raster scan protocol with $512 \times 128$ A scans, 3D imaging data of the $6 \times 6-\mathrm{mm}^{2}$ area were acquired. By weighted moving average, each image had quality sufficient to allow delineation of both the outer border of the RPE and the chorioscleral border, even in eyes with retinal pathology (middle and right, Figure 1; Supplemental Videos 1 and 2). Based on 128 images of the 3D-data set, a choroidal thickness map of the $6 \times 6-\mathrm{mm}^{2}$ area centered on the fovea was created for each eye. In the current study, the mean choroidal thickness in the macula (within the outer circle of the ETDRS grid) was $218.8 \pm$ $69.2 \mu \mathrm{m}$ in Group 1, $218.9 \pm 46.8 \mu \mathrm{m}$ in Group 2, $119.7 \pm 49.2 \mu \mathrm{m}$ in Group 3, $140.1 \pm$ $64.9 \mu \mathrm{m}$ in Group 4, and $144.0 \pm 52.6 \mu \mathrm{m}$ in Group 5. Mean macular choroidal thickness in Group 2 was as great as that in Group 1 (Figure 3). However, mean macular choroidal thickness in Groups 3-5 was significantly less than that in Group $1(P<.05$, respectively) (Figure 4). There were no statistical differences between Groups 3-5.

\section{CHOROIDAL VOLUME}

Macular choroidal volume (within the outer circle of the ETDRS grid) was $6.183 \pm 1.957$ $\mathrm{mm}^{3}$ in Group 1, $6.185 \pm 1.323 \mathrm{~mm}^{3}$ in Group 2, $3.381 \pm 1.389 \mathrm{~mm}^{3}$ in Group 3, $3.959 \pm$ $1.853 \mathrm{~mm}^{3}$ in Group 4, and $4.070 \pm 1.487 \mathrm{~mm}^{3}$ in Group 5. In Groups 3-5, choroidal volume of the macula was significantly reduced compared with that of Group $1(P<.05$, respectively). There were no statistical differences between Groups 3-5. Table 2 shows the choroidal volume in each sector of the ETDRS grid. In most sectors, choroidal volume in Groups 3-5 was significantly less than that of Group 1, respectively.

\section{REGIONAL CHOROIDAL THICKNESS}

Table 3 shows the mean choroidal thickness of each area of the ETDRS grid in the five groups. Mean choroidal thickness within the central area was $238.7 \pm 75.0 \mu \mathrm{m}$ in Group 1, $239.0 \pm 45.4 \mu \mathrm{m}$ in Group 2, $117.4 \pm 55.9 \mu \mathrm{m}$ in Group 3, $144.1 \pm 69.8 \mu \mathrm{m}$ in Group 4, 
and $130.5 \pm 52.8 \mu \mathrm{m}$ in Group 5. However, in these choroidal thickness maps, there were regional irregularities in most eyes (Figures 3 and 4). In all groups, the choroid in the nasal quadrant was thinner than in other quadrants; the mean thickness of the outer nasal sector was significantly less than that of the other sectors $(P<.05$, respectively).

\section{DISCUSSION}

Enhanced depth imaging OCT is coupled usually with multiple averaging in order to produce high contrast images with low speckle noise. ${ }^{16}$ In the studies that have utilized the enhanced depth imaging technique, it was difficult to provide as many sections as does 3D imaging and the choroidal thickness was measured only at the foveal center or, in some cases, at several measurement points. ${ }^{20,21,38-42}$ However, because the choroid often shows focal thickening or thinning, measurement at a single point may be influenced by such irregularity or even by focal indistinctness of the chorioscleral border. ${ }^{11,18,43}$ In the current study, the macular area of eyes with angioid streaks was examined with a raster scan protocol using a prototype swept source OCT, which operated at $1 \mu \mathrm{m}$ wavelength region. Swept source OCT at this longer wavelength has higher penetration and lower scattering at the RPE, and thus allows for full-depth imaging of the choroid. ${ }^{36}$ The tunable laser source in the swept source OCT has a lower signal decay versus depth than does the existing spectral-domain OCT system. In addition, high-speed scanning coupled with the high sensitivity of the swept source OCT allowed us to obtain highly reproducible measurements of the choroidal thickness using a 3D raster scan protocol. Averaging of thickness values obtained at multiple measurement points in the 3D data set contributed to lessening of the error in determining choroidal thickness in the macular area. ${ }^{26,36}$

In the current study, the choroid of eyes with angioid streaks without CNV was as thick as was that in normal controls. Multi-averaged scans of the current OCT system often show a layer of medium diameter blood vessels (Sattler's layer) and an outermost layer of the choroid consisting of larger diameter blood vessels (Haller's layer). ${ }^{34}$ However, the choroid was significantly thinner in eyes with angioid streaks that had developed CNV. Because the configuration of choroidal vessels is disorganized, it is difficult to determine which layer was primarily degenerated. Furthermore, because this study was performed on a cross-sectional basis, the cause of this finding is uncertain, but several hypotheses can be proposed.

First, eyes with angioid streaks may show progressive thinning of the choroid over time. This thinning becomes most evident at a later stage of the disease, at which time many eyes also show secondary CNV. Second, a decrease in choroidal thickness may 
actually contribute to the development of CNV. It is possible that eyes with angioid streaks have a wide range of choroidal thicknesses, and that eyes with a thin choroid develop CNV, while eyes with a more preserved choroid rarely do. Previous histopathologic reports ${ }^{4,44,45}$ have shown that angioid streaks are accompanied by atrophic degeneration of the overlying RPE and by focal breaks or even an absence of the underlying choriocapillaris. These histopathologic changes, which might be due primarily to thickening of the choroidal capillary walls or, more probably, obliteration of their lumens, may be associated with development of CNV. Third, the atrophy of RPE and choriocapillaris that is often seen around the CNV may be associated with the macular choroidal thinning in eyes with CNV secondary to angioid streaks.

In the current study, the choroidal thinning was seen in eyes with CNV, independent of a history of treatments for CNV. However, it is possible that previous treatments for CNV are also involved in the choroidal thinning. Maruko and associates ${ }^{20,42}$ reported recently that subfoveal choroidal thickness is decreased after PDT in eyes with polypoidal choroidal vasculopathy or central serous chorioretinopathy. However, it has also been reported that the subfoveal choroidal thickness is increased during the active phase of these diseases, ${ }^{11,39}$ so it is possible that PDT does not make the choroid pathologically thin by its direct photoreactive effect on the choroidal vessels, but, rather, only reduces the increased subfoveal choroidal thickness to physiologic levels by the reduction of the choroidal hyperpermeability. In our patients treated with PDT (Group 5), choroidal thickness was significantly less, not only in the macular area, where the PDT had been previously performed, but also beyond the vascular arcade. The choroid in eyes that had developed CNV associated with angioid streaks was significantly thinner than was that of normal eyes.

Anti-VEGF treatments for CNV may also be involved in the thinner choroid in Groups 4 and 5. In a computerized search of PubMed, however, we could find no reference to a reduction of choroidal thickness due to anti-VEGF treatment. Recently, Koizumi and associates reported that in eyes with exudative AMD treated with ranibizumab, the mean subfoveal choroidal thickness decreased from $244 \mu \mathrm{m}$ at the baseline to $226 \mu \mathrm{m}$ at 3 months, $229 \mu \mathrm{m}$ at 6 months, and $226 \mu \mathrm{m}$ at 12 months $(P<.01)$ (The 2011 Annual Meeting of American Academy of Ophthalmology, Orlando, FL, October 22-25, 2011). Although the authors found a statistically significant decrease in the foveal choroidal thickness, the mean reduction was only $18 \mu \mathrm{m}$. Based on this evidence, the effect of either PDT or anti-VEGF treatments on the choroidal thickness in our patients would be limited. Previous treatments for CNV do not explain sufficiently the decrease in macular choroidal thickness in eyes with CNV secondary to angioid streaks.

The current results have shown the asymmetric nature of macular choroidal 
thickness not only in normal controls but in eyes with angioid streaks. In our subjects, the choroid in the nasal quadrant was thinner than in other quadrants; this thinning was most evident in the outer nasal sector. The reason for nasal choroidal thinning is unknown, but it may be related to the vascular bed distribution of the choroid and the choroidal watershed zone, which is frequently observed between the macula and optic disc. ${ }^{46}$ In eyes with angioid streaks, secondary CNV occurs usually adjacent to angioid streaks, sometimes in the peripapillary region. ${ }^{3,4}$ Angioid streaks per se may be essential to the development of CNV. In addition, this thinning of nasal choroid might be related to the frequent occurrence of CNV secondary to angioid streaks at this site. However, while eyes with high myopia often show an extremely thin choroid, especially in the nasal quadrant of the macula, myopic CNV is seen usually around the fovea, rarely in the peripapillary area. In addition, all such eyes with a thin choroid do not show myopic CNV. Recently, choroidal thinning has been reported in eyes with exudative AMD, ${ }^{11}$ but so far, the clinical significance of choroidal thinning in the development of CNV has been insufficiently elucidated. ${ }^{47}$

One of the major limitations of the current study is the small sample size in each study group. Unexpectedly, the macular choroidal thickness in Group 3 was slightly thinner than that in Groups 4 and 5, although the difference was not statistically significant $(P=.956$ and $P=.903)$. This difference could be explained by the variability of the macular choroidal thickness in eligible eyes, because the sample size of Group 3 was only 7. Due to this limitation, we could not precisely estimate the effect of previous treatments for CNV on the choroidal thickness. Another limitation of the current study is that the line of RPE and the chorioscleral border were determined manually. The raster scan protocol with over 60,000 measurement points would have minimized any error in measurement made by the observers, so software to determine these lines automatically is essential to further standardize this technique.

In spite of these limitations, our study did show that swept source OCT at $1 \mu \mathrm{m}$ allows us to comprehensively evaluate the macular choroid in eyes with angioid streaks. The choroid in eyes without CNV secondary to angioid streaks was as thick as was that in normal controls, although the choroid was significantly thinner in eyes with angioid streaks that had developed CNV. In addition, the current study confirmed the asymmetric nature of the choroid in the macular area in eyes with angioid streaks. However, as this was a cross-sectional study, various interpretations of our findings are possible and it is difficult to determine whether choroidal thinning is a cause or a consequence of the development of CNV. Additional longitudinal prospective studies are needed to elucidate the role played by the choroid in the development of CNV in eyes with angioid streaks secondary to pseudoxanthoma elasticum. 


\section{ACKNOWLEDGMENTS}

A. Funding/Support: This study was supported in part by the Japan Society for the Promotion of Science (JSPS), Tokyo, Japan (Grant-in-Aid for Scientific Research, no. 21592256), and by the Japan National Society for the Prevention of Blindness, Tokyo, Japan.

B. Financial Disclosure: none.

C. Contributions of Authors: Conception and design of the study (AAE, AT, AM, NY); analysis and interpretation (AAE, AT, AM, KO, MH, SO, KY, MA, NY); writing of the article (AAE, AT); critical revision of the article (AM, KO, MH, SO, KY, MA, NY); final approval of the article (AAE, AT, AM, KO, MH, SO, KY, MA, NY); data collection (AAE, AT, AM, MH, $\mathrm{SO}, \mathrm{KY})$.

D. Ethics Committee Approval: The Institutional Review Board and Ethics Committee of Kyoto University approved this study, which adhered to the tenets of the Declaration of Helsinki. Written informed consent for research participation was obtained from each subject before examination.

E. Other Acknowledgments: none. 


\section{REFERENCES}

1. Le Saux O, Urban Z, Tschuch C, et al. Mutations in a gene encoding an ABC transporter cause pseudoxanthoma elasticum. Nat Genet 2000;25(2):223-227.

2. Finger RP, Charbel Issa P, Ladewig MS, et al. Pseudoxanthoma elasticum: genetics, clinical manifestations and therapeutic approaches. Surv Ophthalmol 2009;54(2):272-285.

3. Clarkson JG, Altman RD. Angioid streaks. Surv Ophthalmol 1982;26(5):235-246.

4. Dreyer R, Green WR. The pathology of angioid streaks: a study of twenty-one cases. Trans Pa Acad Ophthalmol Otolaryngol 1978;31(2):158-167.

5. Mimoun G, Tilleul J, Leys A, Coscas G, Soubrane G, Souied EH. Intravitreal ranibizumab for choroidal neovascularization in angioid streaks. Am J Ophthalmol 2010;150(5):692-700.

6. Myung JS, Bhatnagar P, Spaide RF, et al. Long-term outcomes of intravitreal antivascular endothelial growth factor therapy for the management of choroidal neovascularization in pseudoxanthoma elasticum. Retina 2010;30(5):748-755.

7. Connor PJ Jr, Juergens JL, Perry HO, Hollenhorst RW, Edwards JE. Pseudoxanthoma elasticum and angioid streaks. A review of 106 cases. Am J Med .21961;30:537-543.

8. Shaikh S, Ruby AJ, Williams GA. Photodynamic therapy using verteporfin for choroidal neovascularization in angioid streaks. Am J Ophthalmol 2003;135(1):1-6.

9. Mennel S, Schmidt JC, Meyer $\mathrm{CH}$. Therapeutic strategies in choroidal neovascularizations secondary to angioid streaks. Am J Ophthalmol 2003;136(3):580-582.

10. Linsenmeier RA, Padnick-Silver L. Metabolic dependence of photoreceptors on the choroid in the normal and detached retina. Invest Ophthalmol Vis Sci 2000;41(10):3117-3123.

11. Chung SE, Kang SW, Lee JH, Kim YT. Choroidal thickness in polypoidal choroidal vasculopathy and exudative age-related macular degeneration. Ophthalmology 2011;118(5):840-845.

12. Gomi F, Tano Y. Polypoidal choroidal vasculopathy and treatments. Curr Opin Ophthalmol 2008;19(3):208-212.

13. Spaide RF, Hall L, Haas A, et al. Indocyanine green videoangiography of older patients with central serous chorioretinopathy. Retina 1996;16(3):203-213.

14. Fong $\mathrm{AH}$, Li KK, Wong $\mathrm{D}$. Choroidal evaluation using enhanced depth imaging spectral-domain optical coherence tomography in Vogt-Koyanagi-Harada disease. Retina 2011;31(3):502-509. 
15. Fujiwara T, Imamura Y, Margolis R, Slakter JS, Spaide RF. Enhanced depth imaging optical coherence tomography of the choroid in highly myopic eyes. Am J Ophthalmol 2009;148(3):445-450.

16. Spaide RF, Koizumi H, Pozzoni MC. Enhanced depth imaging spectral-domain optical coherence tomography. Am J Ophthalmol 2008;146(4):496-500.

17. van Velthoven ME, Faber DJ, Verbraak FD, van Leeuwen TG, de Smet MD. Recent developments in optical coherence tomography for imaging the retina. Prog Retin Eye Res 2007;26(1):57-77.

18. Koizumi H, Yamagishi T, Yamazaki T, Kawasaki R, Kinoshita S. Subfoveal choroidal thickness in typical age-related macular degeneration and polypoidal choroidal vasculopathy. Graefes Arch Clin Exp Ophthalmol 2011;249(8):1123-1128.

19. Margolis R, Spaide RF. A pilot study of enhanced depth imaging optical coherence tomography of the choroid in normal eyes. Am J Ophthalmol 2009;147(5):811-815.

20. Maruko I, lida T, Sugano Y, et al. Subfoveal choroidal thickness after treatment of Vogt-Koyanagi-Harada disease. Retina 2011;31(3):510-517.

21. Reibaldi M, Boscia F, Avitabile T, et al. Enhanced depth imaging optical coherence tomography of the choroid in idiopathic macular hole: A cross-sectional prospective study. Am J Ophthalmol 2011;151(1):112-117.

22. Spaide RF. Age-related choroidal atrophy. Am J Ophthalmol 2009;147(5):801-810.

23. Yeoh J, Rahman W, Chen F, et al. Choroidal imaging in inherited retinal disease using the technique of enhanced depth imaging optical coherence tomography. Graefes Arch Clin Exp Ophthalmol 2010;248(12):1719-1728.

24. Blackburn J, McGwin G Jr. Enhanced depth imaging optical coherence tomography of the choroid in idiopathic macular hole. Am J Ophthalmol 2011;151(3):560-561.

25. Imamura Y, lida T, Maruko I, Zweifel SA, Spaide RF. Enhanced depth imaging optical coherence tomography of the sclera in dome-shaped macula. Am J Ophthalmol 2011;151(2):297-302.

26. Agawa T, Miura M, Ikuno Y, et al. Choroidal thickness measurement in healthy Japanese subjects by three-dimensional high-penetration optical coherence tomography. Graefes Arch Clin Exp Ophthalmol 2011;249(10)1485-1492.

27. Ikuno Y, Kawaguchi K, Nouchi T, Yasuno Y. Choroidal thickness in healthy Japanese subjects. Invest Ophthalmol Vis Sci 2010;51(4):2173-2176.

28. Ikuno Y, Maruko I, Yasuno Y, et al. Reproducibility of retinal and choroidal thickness measurements in enhanced depth imaging and high-penetration optical coherence tomography. Invest Ophthalmol Vis Sci 2011;52(8):5536-5540.

29. de Bruin DM, Burnes DL, Loewenstein J, et al. In vivo three-dimensional imaging of neovascular age-related macular degeneration using optical frequency domain 
imaging at $1050 \mathrm{~nm}$. Invest Ophthalmol Vis Sci 2008;49(10):4545-4552.

30. Yasuno $Y$, Hong Y, Makita S, et al. In vivo high-contrast imaging of deep posterior eye by 1-micron swept source optical coherence tomography and scattering optical coherence angiography. Opt Express 2007;15(10):6121-6139.

31. Esmaeelpour M, Povazay B, Hermann B, et al. Mapping choroidal and retinal thickness variation in type 2 diabetes using three-dimensional 1060-nm optical coherence tomography. Invest Ophthalmol Vis Sci 2011;52(8):5311-5316.

32. Esmaeelpour M, Povazay B, Hermann B, et al. Three-dimensional 1060-nm OCT: choroidal thickness maps in normal subjects and improved posterior segment visualization in cataract patients. Invest Ophthalmol Vis Sci 2010;51(10):5260-5266.

33. Wood A, Binns A, Margrain T, et al. Retinal and choroidal thickness in early age-related macular degeneration. Am J Ophthalmol 2011;152(6):1030-1038.

34. Povazay B, Hermann B, Hofer B, et al. Wide-field optical coherence tomography of the choroid in vivo. Invest Ophthalmol Vis Sci 2009;50(4):1856-1863.

35. Shields JA, Federman JL, Tomer TL, Annesley WH Jr. Angioid streaks. I. Ophthalmoscopic variations and diagnostic problems. Br J Ophthalmol 1975;59(5):257-266.

36. Hirata M, Tsujikawa A, Matsumoto A, et al. Macular choroidal thickness and volume in normal subjects measured by swept-source optical coherence tomography. Invest Ophthalmol Vis Sci 2011;52(8):4971-4978.

37. Early Treatment Diabetic Retinopathy Study research group. Photocoagulation for diabetic macular edema. Early Treatment Diabetic Retinopathy Study report number 1. Arch Ophthalmol 1985;103(12):1796-1806.

38. Spaide RF. Enhanced depth imaging optical coherence tomography of retinal pigment epithelial detachment in age-related macular degeneration. Am J Ophthalmol 2009;147(4):644-652.

39. Imamura Y, Fujiwara T, Margolis R, Spaide RF. Enhanced depth imaging optical coherence tomography of the choroid in central serous chorioretinopathy. Retina 2009;29(10):1469-1473.

40. Maruko I, lida T, Sugano Y, Ojima A, Ogasawara M, Spaide RF. Subfoveal choroidal thickness after treatment of central serous chorioretinopathy. Ophthalmology 2010;117(9):1792-1799.

41. Vance SK, Imamura Y, Freund KB. The effects of sildenafil citrate on choroidal thickness as determined by enhanced depth imaging optical coherence tomography. Retina 2011;31(2):332-335.

42. Maruko I, lida T, Sugano Y, Saito M, Sekiryu T. Subfoveal retinal and choroidal thickness after verteporfin photodynamic therapy for polypoidal choroidal 
vasculopathy. Am J Ophthalmol 2011;151(4):594-603.

43. Rahman W, Chen FK, Yeoh J, Patel P, Tufail A, Da Cruz L. Repeatability of manual subfoveal choroidal thickness measurements in healthy subjects using the technique of enhanced depth imaging optical coherence tomography. Invest Ophthalmol Vis Sci 2011;52(5):2267-2271.

44. Gass JDM, Clarkson JG. Angioid streaks and disciform macular detachment in Pagets disease (osteitis deformans). Am J Ophthalmol 1973;75(4):576-586.

45. Walker ER, Frederickson RG, Mayes MD. The mineralization of elastic fibers and alterations of extracellular matrix in pseudoxanthoma elasticum. Ultrastructure, immunocytochemistry, and X-ray analysis. Arch Dermatol 1989;125(1):70-76.

46. Hayreh SS. In vivo choroidal circulation and its watershed zones. Eye (Lond) 1990;4 ( Pt 2):273-289.

47. Nickla DL, Wallman J. The multifunctional choroid. Prog Retin Eye Res 2010;29(2):144-168. 


\section{Figure Captions}

Figure 1. Images of a healthy subject obtained with swept source optical coherence tomography (OCT) at 1,050 nm. (Left) A multi-averaged horizontal OCT image of $12 \mathrm{~mm}$ in length was obtained by averaging 50 images, which consisted of 1,024 A scans. Fine structure from the inner limiting membrane to the chorioscleral border is seen clearly beyond the vascular arcade. Regional changes in choroidal thickness are seen. (Middle and right) In a raster scan protocol, 128 consecutive images, consisting of 512 A-scans, were obtained. To reduce speckle noise, each image was enhanced by weighted moving average of three consecutive original images. (Middle) Single OCT image acquired with a raster scan protocol without weighted moving average. (Right) Single OCT image acquired with a raster scan protocol with weighted moving average.

Figure 2. Multi-averaged horizontal section through the fovea obtained by swept source optical coherence tomography (left) and fundus photograph (right) in a healthy eye and in eyes with angioid streaks. (Top) A healthy eye (Group 1). Choroidal structures and the chorioscleral border are clearly seen. The choroid seems to be thin around the optic disc. (2nd row) An eye with angioid streaks without choroidal neovascularization (CNV) (Group 2). The choroidal thickness is well preserved. (3rd row) An eye with CNV secondary to angioid streaks that had no history of treatments (Group 3). Choroidal thickness is substantially reduced in the entire macular area. (4th row) An eye with CNV secondary to angioid streaks that had previously received only anti-vascular endothelial growth factor treatments with no history of photodynamic therapy (PDT) (Group 4). Choroidal thickness is substantially reduced in the entire macular area. Regional thickening of the choroid is seen on the temporal side (arrow). (Bottom) An eye with CNV secondary to angioid streaks that was treated previously with PDT (Group 5). Choroidal thickness is reduced not only in the PDT-treated macular area but also beyond the vascular arcade.

Figure 3. Choroidal thickness map of an eye with angioid streaks that had no choroidal neovascularization (group 2). (Top left) Fundus photograph of the right eye of a 68-year-old man; visual acuity was $20 / 20$. (Top right) Fluorescein angiogram shows only a window defect. (Middle) A multi-averaged horizontal image obtained with swept source optical coherence tomography clearly shows choroidal structures and the chorioscleral border. The choroid appears to have a physiologic thickness. (Bottom left) Choroidal thickness map of $6 \times 6-\mathrm{mm}^{2}$ of the macular area. Choroid in the nasal quadrant is thinner than is that of other quadrants. In addition, there is regional thickening in the outer superior area and in the outer inferotemporal area. (Bottom right) By applying the ETDRS 
grid to the map, mean choroidal thickness was obtained for each sector.

Figure 4. Choroidal thickness map of an eye with choroidal neovascularization (CNV) associated with angioid streaks. (Top left) Fundus photograph of the left eye of an 80 -year-old woman; visual acuity was $20 / 30$. This eye was previously treated with one intravitreal injection of bevacizumab and 3 injections of ranibizumab (Group 4). (Top right) Fluorescein angiogram shows occult choroidal neovascularization. (Middle) $A$ multi-averaged horizontal image obtained with swept source optical coherence tomography shows the thin choroid with type 1 choroidal neovascularization. (Bottom left) Choroidal thickness map of a $6 \times 6-\mathrm{mm}^{2}$ of the macular area. Choroidal thickness is reduced in the entire macular area. (Bottom right) By applying the ETDRS grid to the map, mean choroidal thickness was obtained for each area. 
Macular Choroidal Thickness in AS. Ellabban AA, et al.

TABLE 1. Characteristics of Eyes and Mean Choroidal Thickness and Volume Obtained by Swept Source Optical Coherence Tomography

\begin{tabular}{|c|c|c|c|c|c|c|}
\hline & Group 1 & Group 2 & Group 3 & Group 4 & Group 5 & \\
\hline & Normal eyes & $\begin{array}{c}\text { AS without } \\
\text { CNV }\end{array}$ & $\begin{array}{l}\text { AS with CNV } \\
\text { and no history } \\
\text { of treatment }\end{array}$ & $\begin{array}{l}\text { AS with CNV } \\
\text { and a history of } \\
\text { only anti-VEGF } \\
\text { treatments }\end{array}$ & $\begin{array}{l}\text { AS with CNV } \\
\text { and a history } \\
\text { of PDT }\end{array}$ & $\begin{array}{c}P \\
\text { value }\end{array}$ \\
\hline Number of eyes & 20 & 6 & 7 & 11 & 15 & \\
\hline Gender (male/female) & $10 / 10$ & $4 / 2$ & $4 / 3$ & $8 / 3$ & $8 / 7$ & \\
\hline Age (years) & $67.4 \pm 13.2$ & $66.0 \pm 6.8$ & $65.1 \pm 7.1$ & $65.6 \pm 7.8$ & $66.7 \pm 9.9$ & .960 \\
\hline Axial length (mm) & $23.5 \pm 1.3$ & $23.3 \pm 1.0$ & $23.6 \pm 1.1$ & $23.9 \pm 1.5$ & $23.4 \pm 1.8$ & .949 \\
\hline Refractive error (diopters) & $-0.51 \pm 2.96$ & $0.01 \pm 1.02$ & $0.11 \pm 1.93$ & $-1.38 \pm 2.50$ & $-2.20 \pm 1.90$ & .134 \\
\hline Visual acuity (logMAR) & $0.01 \pm 0.21$ & $-0.14 \pm 0.05$ & $1.02 \pm 0.32^{*}$ & $0.45 \pm 0.61^{*}$ & $0.80 \pm 0.45^{\star}$ & $<.001$ \\
\hline Foveal retinal thickness $(\mu \mathrm{m})$ & $204.4 \pm 35.5$ & $194.0 \pm 12.6$ & $260.3 \pm 119.5$ & $209.9 \pm 97.8$ & $154.4 \pm 64.3$ & .027 \\
\hline Foveal choroidal thickness $(\mu \mathrm{m})$ & $254.8 \pm 76.0$ & $244.7 \pm 51.0$ & $142.0 \pm 67.9^{*}$ & $144.0 \pm 79.8^{*}$ & $124.8 \pm 54.2^{*}$ & $<.001$ \\
\hline Mean choroidal thickness within a circle of $1.0-\mathrm{mm}$ diameter $(\mu \mathrm{m})$ & $238.7 \pm 75.0$ & $239.0 \pm 45.4$ & $117.4 \pm 55.9^{*}$ & $144.1 \pm 69.8^{\star}$ & $130.5 \pm 52.8^{*}$ & $<.001$ \\
\hline Mean choroidal thickness within a circle of 3.0-mm diameter $(\mu \mathrm{m})$ & $233.0 \pm 72.4$ & $232.7 \pm 49.7$ & $116.5 \pm 52.4^{*}$ & $139.0 \pm 68.9^{*}$ & $137.7 \pm 57.6^{*}$ & $<.001$ \\
\hline Mean choroidal thickness within a circle of $6.0-\mathrm{mm}$ diameter $(\mu \mathrm{m})$ & $218.8 \pm 69.2$ & $218.9 \pm 46.8$ & $119.7 \pm 49.2^{*}$ & $140.1 \pm 64.9^{\dagger}$ & $144.0 \pm 52.6^{\dagger}$ & $<.001$ \\
\hline Choroidal volume within a circle of $1.0-\mathrm{mm}$ diameter $\left(\mathrm{mm}^{3}\right)$ & $0.187 \pm 0.059$ & $0.188 \pm 0.036$ & $0.092 \pm 0.044^{*}$ & $0.113 \pm 0.056^{*}$ & $0.102 \pm 0.041^{*}$ & $<.001$ \\
\hline Choroidal volume within a circle of $3.0-\mathrm{mm}$ diameter $\left(\mathrm{mm}^{3}\right)$ & $1.646 \pm 0.512$ & $1.644 \pm 0.351$ & $0.823 \pm 0.370^{*}$ & $0.982 \pm 0.487^{*}$ & $0.973 \pm 0.407^{*}$ & $<.001$ \\
\hline Choroidal volume within a circle of $6.0-\mathrm{mm}$ diameter $\left(\mathrm{mm}^{3}\right)$ & $6.183 \pm 1.957$ & $6.185 \pm 1.323$ & $3.381 \pm 1.389^{*}$ & $3.959 \pm 1.853^{\dagger}$ & $4.070 \pm 1.487^{\dagger}$ & $<.001$ \\
\hline CNV (active/inactive) & - & - & $4 / 3$ & $5 / 6$ & $1 / 14$ & \\
\hline \multicolumn{7}{|l|}{ Treatment } \\
\hline Number of anti-VEGF treatments & 0 & 0 & 0 & $3.4 \pm 2.1$ & $3.1 \pm 3.9$ & \\
\hline Number of PDT treatments & 0 & 0 & 0 & 0 & $2.9 \pm 1.9$ & \\
\hline
\end{tabular}

AS = angioid streaks; CNV = choroidal neovascularization; PDT = photodynamic therapy; logMAR = logarithm of minimal angle of resolution; VEGF = vascular endothelial growth factor. ${ }^{\star} P<.01,{ }^{\dagger} P<.05$, compared with Group 1. 
TABLE 2. Choroidal Volume in Each Sector of ETDRS Grid Obtained with Swept Source Optical Coherence Tomography

\begin{tabular}{|c|c|c|c|c|c|}
\hline \multirow[b]{3}{*}{ Sector } & \multicolumn{5}{|c|}{ Mean choroidal volume $\left(\mathrm{mm}^{3}\right)$} \\
\hline & Group 1 & Group 2 & Group 3 & Group 4 & Group 5 \\
\hline & Normal eyes & AS without CNV & $\begin{array}{l}\text { AS with CNV and no } \\
\text { history of treatments }\end{array}$ & $\begin{array}{l}\text { AS with CNV and a } \\
\text { history of only } \\
\text { anti-VEGF treatments }\end{array}$ & $\begin{array}{c}\text { AS with CNV and a } \\
\text { history of } \\
\text { photodynamic therapy }\end{array}$ \\
\hline Central area $\left(P\right.$ value $\left.{ }^{*}\right)$ & $0.187 \pm 0.059$ & $0.188 \pm 0.036(1.000)$ & $0.092 \pm 0.043(.001)$ & $0.113 \pm 0.056(.002)$ & $0.102 \pm 0.041(<.001)$ \\
\hline Inner superior ( $P$ value $\left.{ }^{*}\right)$ & $0.375 \pm 0.114$ & $0.402 \pm 0.081(.947)$ & $0.186 \pm 0.080(.001)$ & $0.232 \pm 0.119(.005)$ & $0.229 \pm 0.098(.001)$ \\
\hline Inner nasal ( $P$ value $\left.{ }^{*}\right)$ & $0.354 \pm 0.118$ & $0.331 \pm 0.084(.990)$ & $0.150 \pm 0.615(<.001)$ & $0.203 \pm 0.117(.004)$ & $0.164 \pm 0.080(<.001)$ \\
\hline Inner inferior $\left(P\right.$ value $\left.{ }^{*}\right)$ & $0.368 \pm 0.113$ & $0.352 \pm 0.097(.989)$ & $0.182 \pm 0.104(.003)$ & $0.207 \pm 0.108(.002)$ & $0.228 \pm 0.113(.003)$ \\
\hline Inner temporal ( $P$ value $\left.{ }^{*}\right)$ & $0.369 \pm 0.119$ & $0.371 \pm 0.073(1.000)$ & $0.212 \pm 0.092(.007)$ & $0.226 \pm 0.097(.003)$ & $0.249 \pm 0.090(.006)$ \\
\hline Outer superior ( $P$ value $\left.{ }^{*}\right)$ & $1.301 \pm 0.379$ & $1.361 \pm 0.322(.984)$ & $0.753 \pm 0.296(.007)$ & $0.887 \pm 0.422(.018)$ & $0.873 \pm 0.289(.006)$ \\
\hline Outer nasal $\left(P\right.$ value $\left.{ }^{*}\right)$ & $0.939 \pm 0.375$ & $0.826 \pm 0.218(.878)$ & $0.384 \pm 0.192(.002)$ & $0.554 \pm 0.384(.014)$ & $0.473 \pm 0.220(.001)$ \\
\hline Outer inferior ( $P$ value $\left.{ }^{*}\right)$ & $1.111 \pm 0.394$ & $1.173 \pm 0.414(.983)$ & $0.659 \pm 0.300(.046)$ & $0.713 \pm 0.324(.029)$ & $0.805 \pm 0.347(.085)$ \\
\hline Outer temporal ( $P$ value $\left.{ }^{*}\right)$ & $1.185 \pm 0.378$ & $1.197 \pm 0.231(1.000)$ & $0.770 \pm 0.286(.033)$ & $0.806 \pm 0.278(.021)$ & $0.946 \pm 0.280(.139)$ \\
\hline
\end{tabular}

AS = Angioid streaks; CNV = choroidal neovascularization; VEGF = vascular endothelial growth factor; ETDRS = Early Treatment Diabetic Retinopathy Study.

Mean choroidal volumes in each area were compared using one-way analysis of variance with Tukey's post-hoc analysis.

${ }^{*} P$ value, compared with values in Group 1. 
TABLE 3. Mean Choroidal Thickness in Each Sector of the ETDRS Grid Obtained with Swept Source Optical Coherence Tomography

\begin{tabular}{|c|c|c|c|c|c|}
\hline & & \multicolumn{4}{|c|}{ Mean choroidal thickness of inner ring sectors $(\mu \mathrm{m})$} \\
\hline & & Inner superior & Inner nasal & Inner inferior & Inner temporal \\
\hline Group 1 & $\begin{array}{l}\text { Normal eyes } \\
\left.\qquad(P \text { value })^{*}\right)\end{array}$ & $\begin{array}{c}235.5 \pm 75.4 \\
(.006)\end{array}$ & $220.4 \pm 75.0$ & $\begin{array}{c}234.4 \pm 72.2 \\
(.058)\end{array}$ & $\begin{array}{c}235.5 \pm 75.4 \\
(.043)\end{array}$ \\
\hline Group 2 & $\begin{array}{l}\text { AS without CNV } \\
(P \text { value })\end{array}$ & $\begin{array}{l}256.2 \pm 51.6 \\
\quad(.012)\end{array}$ & $210.9 \pm 53.6$ & $\begin{array}{l}224.0 \pm 61.9 \\
(.724)\end{array}$ & $\begin{array}{c}236.5 \pm 46.6 \\
(.217)\end{array}$ \\
\hline Group 3 & $\begin{array}{l}\text { AS with } \mathrm{CNV} \text { and no history of } \\
\left.\text { treatments ( } P \text { value }{ }^{*}\right)\end{array}$ & $\begin{array}{c}118.4 \pm 50.9 \\
(.119)\end{array}$ & $95.7 \pm 39.2$ & $\begin{array}{c}116.0 \pm 66.4 \\
(.186)\end{array}$ & $\begin{array}{c}135.3 \pm 58.5 \\
(.003)\end{array}$ \\
\hline Group 4 & $\begin{array}{l}\text { AS with CNV and a history of only } \\
\text { anti-VEGF treatments }\left(P \text { value }{ }^{*}\right)\end{array}$ & $\begin{array}{c}148.1 \pm 75.9 \\
(.067)\end{array}$ & $129.4 \pm 74.9$ & $\begin{array}{c}132.1 \pm 69.0 \\
(.983)\end{array}$ & $\begin{array}{l}144.0 \pm 62.3 \\
(.202)\end{array}$ \\
\hline \multirow[t]{3}{*}{ Group 5} & $\begin{array}{l}\text { AS with CNV and a history of } \\
\left.\text { photodynamic therapy ( } P \text { value }{ }^{*}\right)\end{array}$ & $\begin{array}{l}145.7 \pm 62.5 \\
\quad(<.001)\end{array}$ & $104.9 \pm 51.2$ & $\begin{array}{l}145.1 \pm 72.2 \\
\quad(<.001)\end{array}$ & $\begin{array}{l}158.7 \pm 57.6 \\
\quad(<.001)\end{array}$ \\
\hline & & \multicolumn{4}{|c|}{ Mean choroidal thickness of outer ring sectors $(\mu \mathrm{m})$} \\
\hline & & Outer superior & Outer nasal & Outer inferior & Outer temporal \\
\hline Group 1 & $\begin{array}{l}\text { Normal eyes } \\
\qquad(P \text { value })^{*}\end{array}$ & $\begin{array}{c}245.6 \pm 71.6 \\
(.001)\end{array}$ & $177.2 \pm 70.9$ & $\begin{array}{c}209.6 \pm 74.5 \\
(<.001)\end{array}$ & $\begin{array}{c}223.6 \pm 73.1 \\
(<.001)\end{array}$ \\
\hline Group 2 & $\begin{array}{l}\text { AS without CNV } \\
\qquad(P \text { value })^{*}\end{array}$ & $\begin{array}{c}256.9 \pm 60.8 \\
(.001)\end{array}$ & $155.9 \pm 41.1$ & $\begin{array}{c}221.4 \pm 75.0 \\
(.031)\end{array}$ & $\begin{array}{c}222.8 \pm 40.2 \\
(.027)\end{array}$ \\
\hline Group 3 & $\begin{array}{l}\text { AS with CNV and no history of } \\
\left.\text { treatments ( } P \text { value }{ }^{*}\right)\end{array}$ & $\begin{array}{l}142.1 \pm 55.9 \\
(<.001)\end{array}$ & $72.5 \pm 36.3$ & $\begin{array}{c}124.4 \pm 56.6 \\
(<.001)\end{array}$ & $\begin{array}{l}145.3 \pm 54.0 \\
(<.001)\end{array}$ \\
\hline Group 4 & $\begin{array}{l}\text { AS with CNV and a history of only } \\
\left.\text { anti-VEGF treatments ( } P \text { value }{ }^{\star}\right)\end{array}$ & $\begin{array}{c}167.5 \pm 79.7 \\
\quad(<.001)\end{array}$ & $104.6 \pm 72.6$ & $\begin{array}{c}134.6 \pm 61.2 \\
(.022)\end{array}$ & $\begin{array}{l}155.1 \pm 51.5 \\
(<.001)\end{array}$ \\
\hline Group 5 & $\begin{array}{l}\text { AS with CNV and a history of } \\
\left.\text { photodynamic therapy ( } P \text { value }{ }^{*}\right)\end{array}$ & $\begin{array}{l}164.9 \pm 54.5 \\
\quad(<.001)\end{array}$ & $89.4 \pm 41.6$ & $\begin{array}{c}152.0 \pm 65.2 \\
(<.001)\end{array}$ & $\begin{array}{l}178.3 \pm 53.0 \\
\quad(<.001)\end{array}$ \\
\hline
\end{tabular}

AS = angioid streaks; CNV = choroidal neovascularization; VEGF = vascular endothelial growth factor; ETDRS = Early Treatment Diabetic Retinopathy Study.

Mean choroidal thickness in each sector was compared using two-way analysis of variance with Tukey's post-hoc analysis.

${ }^{*} P$ value, compared with values in nasal sector. 
Figure 1
Click here to download high resolution image
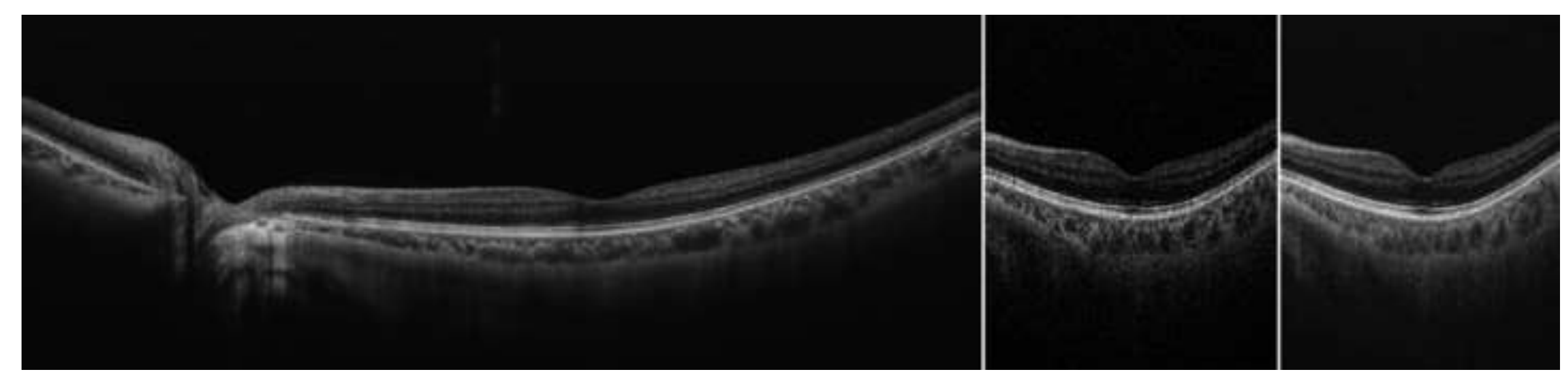


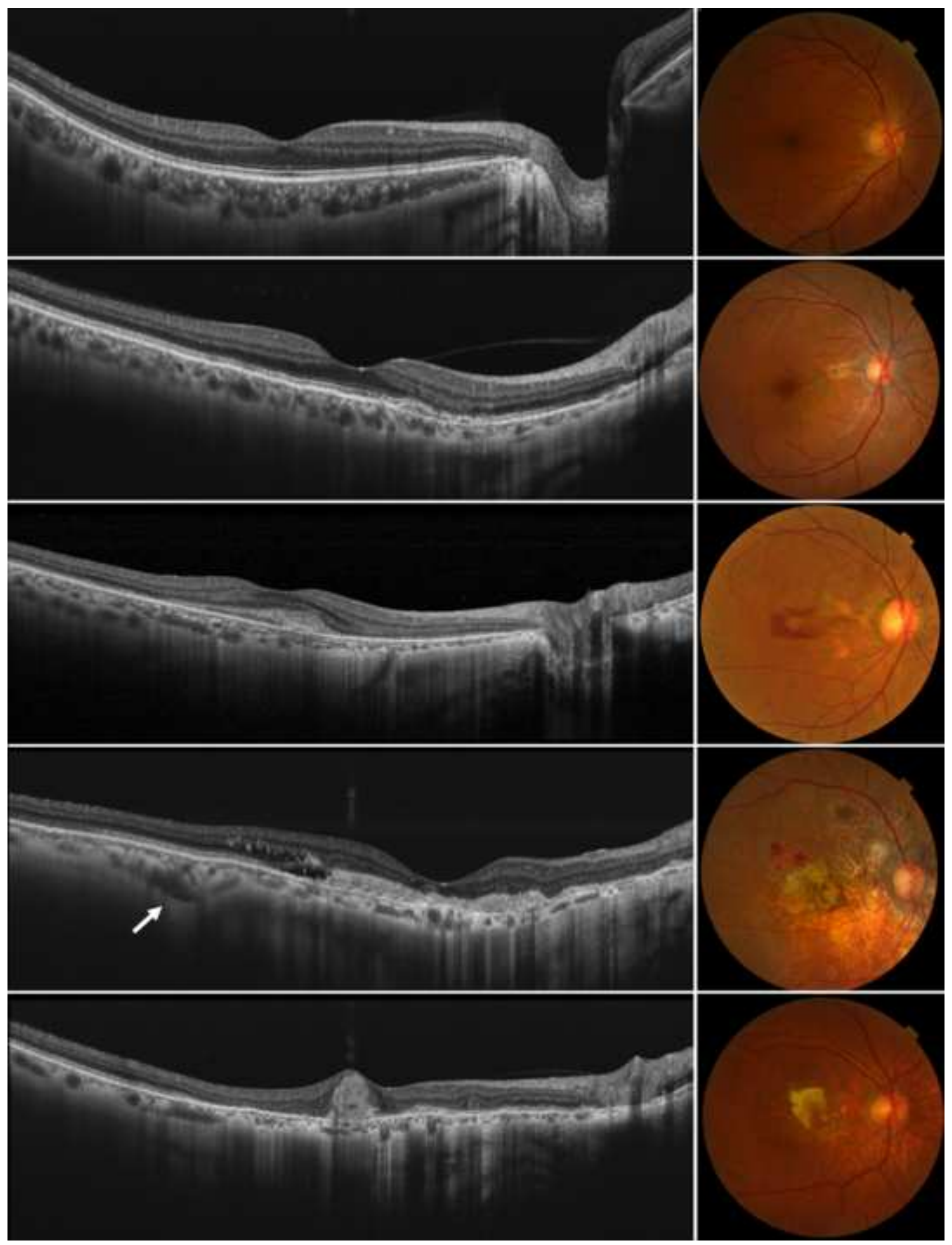




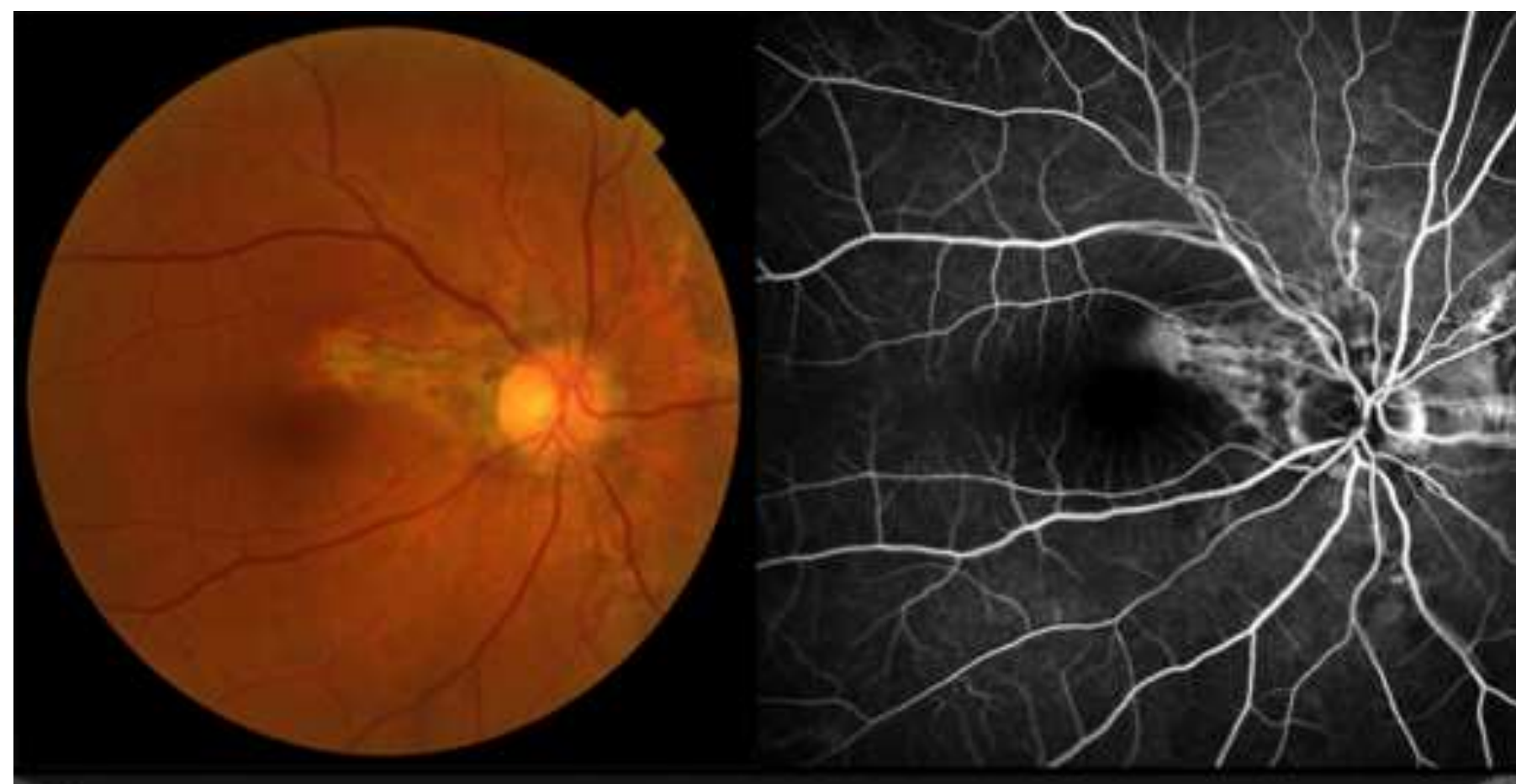

Tempora!

Nasal

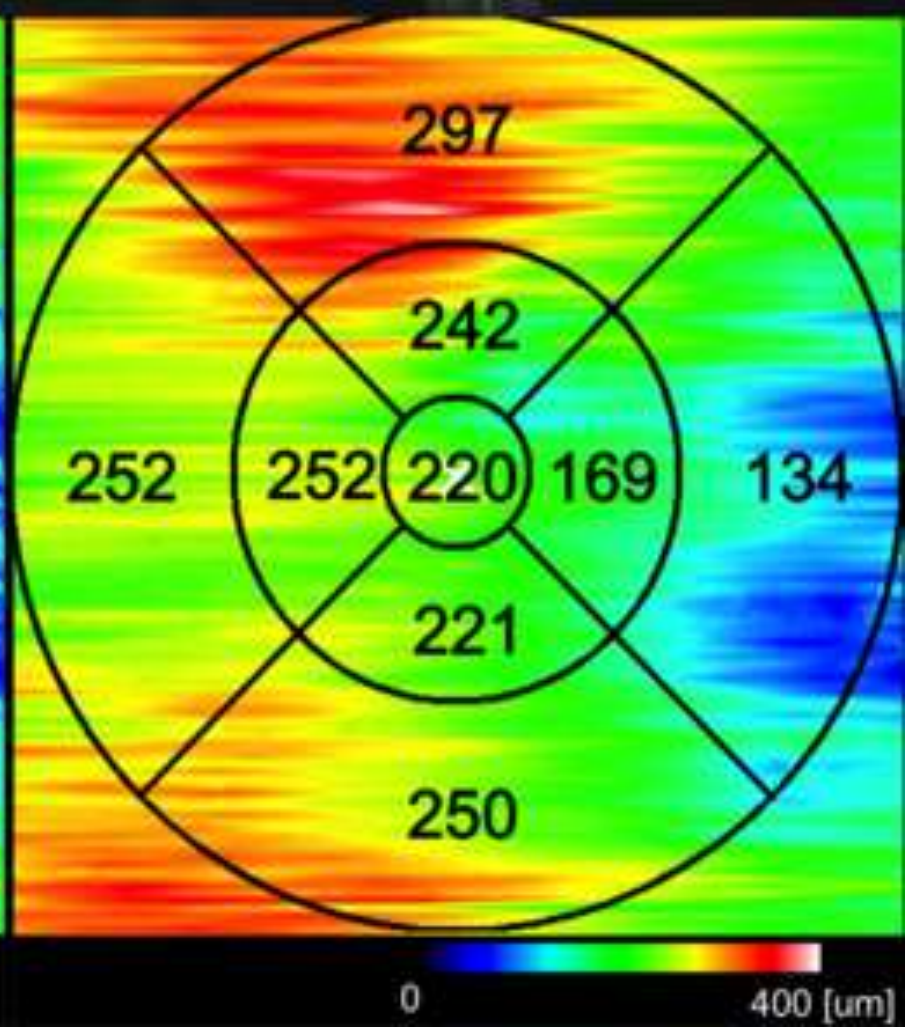




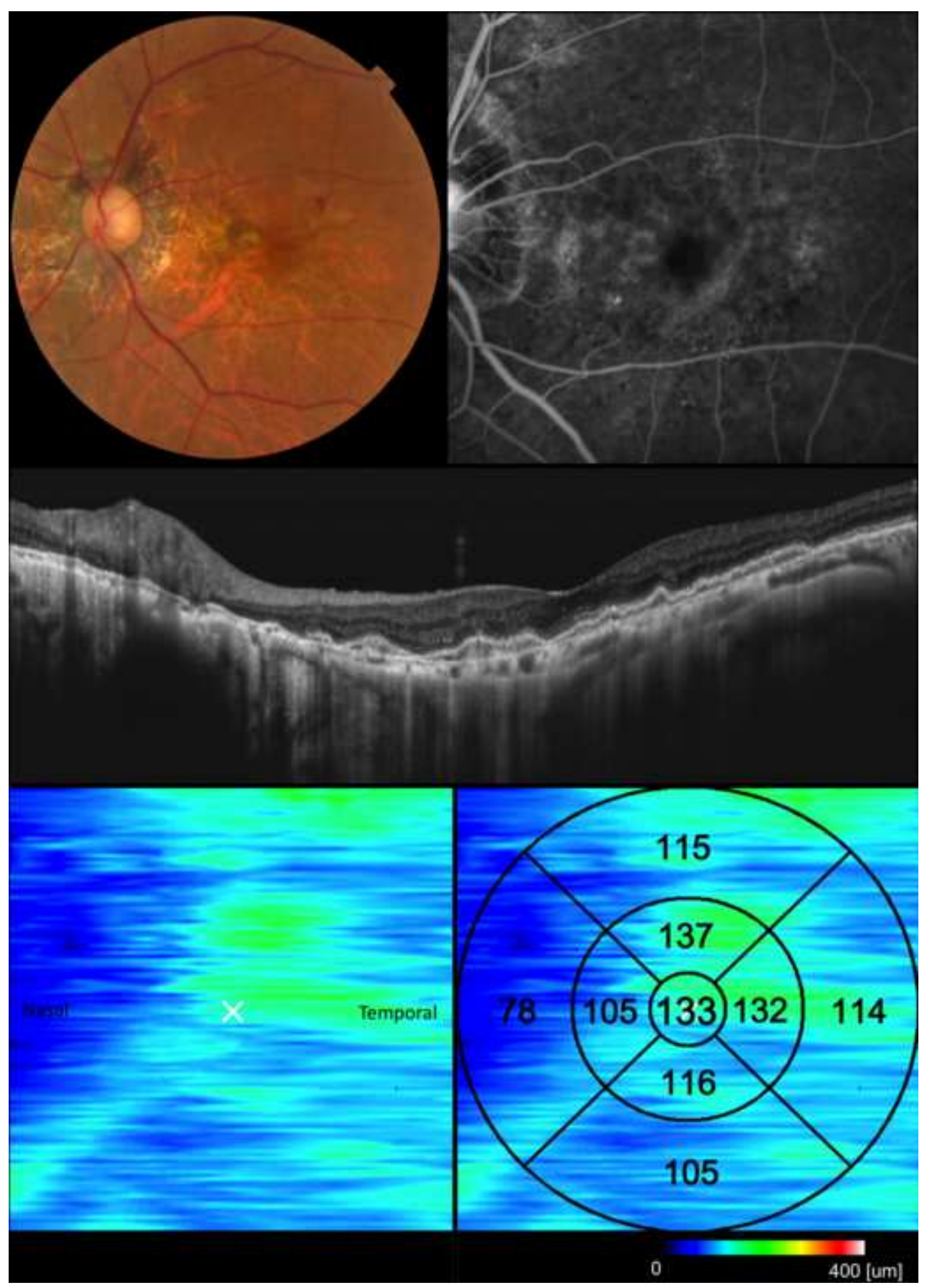




\section{Table of Contents Statement}

Using swept source-optical coherence tomography in the $1050 \mathrm{~nm}$ wavelength range, choroidal thickness of the macula area were examined in eyes with angioid streaks secondary to pseudoxanthoma elasticum. The choroid in eyes with angioid streaks without choroidal neovascularization was as thick as that in normal controls, but was significantly thinner in eyes with angioid streaks that had developed choroidal neovascularization. The choroid of the nasal quadrant was significantly thinner compared to that in other quadrants. 


\section{Biographic sketch}

Abdallah A. Ellabban, M.D., graduated from Suez Canal University (Egypt) and obtained his M.D. in 2003. He completed his residency program and obtained a masters degree in ophthalmology from Suez Canal University in 2007. Dr Abdallah is now in a PhD program in the Department of Ophthalmology and Visual Sciences at Kyoto University (Japan) under the supervision of Professor Nagahisa Yoshimura. 
E-

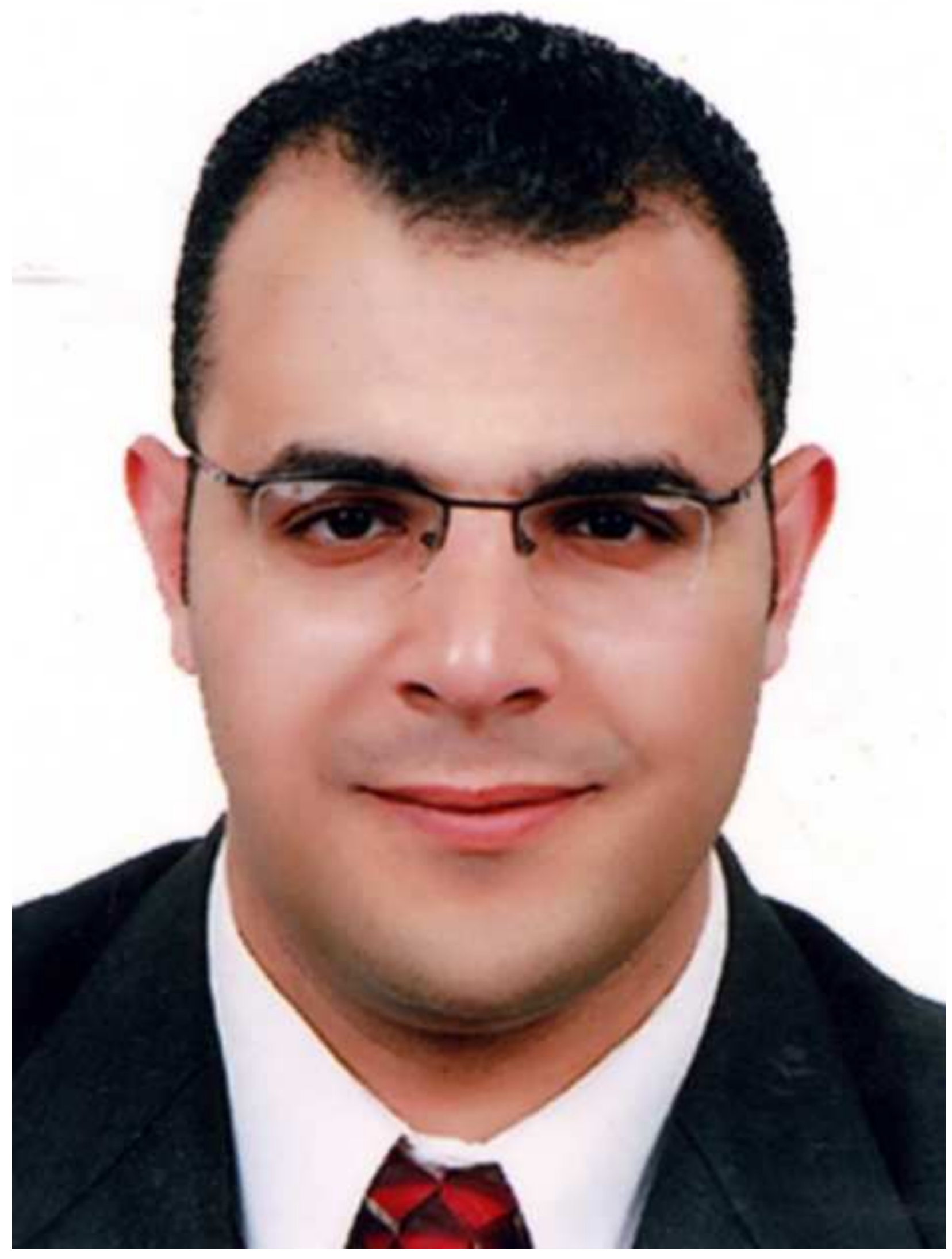




\section{Supplemental Figure Legends}

Supplemental Video 1. A series of images acquired across the macular area of a healthy eye. Images were obtained with swept source optical coherence tomography using a raster scan protocol of 512 (horizontal) x 128 (vertical) A-scans. To reduce speckle noise, each image was enhanced by weighted moving average of three consecutive original images.

Supplemental Video 2. A series of images acquired across the macular area; these images show choroidal neovascularization secondary to angioid streaks. Images were obtained with swept source optical coherence tomography using a raster scan protocol of 512 (horizontal) x 128 (vertical) A-scans. To reduce speckle noise, each image was enhanced by weighted moving average of three consecutive original images. The examined eye was treated six times with an intravitreal injection of ranibizumab. 
S 亲都大学/ideo 1

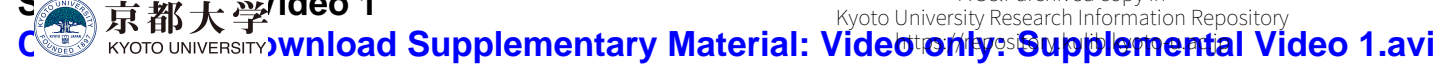
. 
S 京都大学 Video 2 